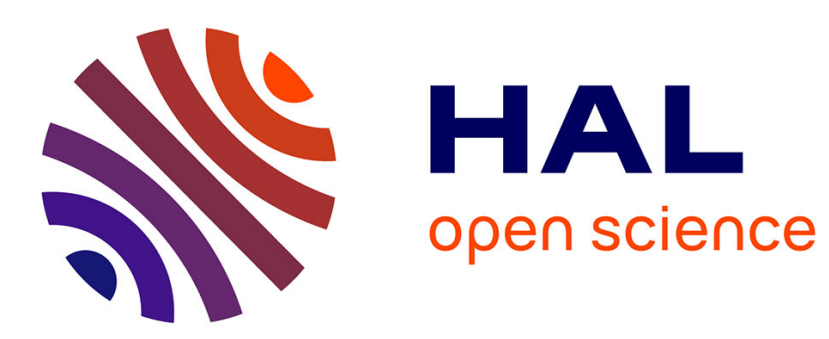

\title{
Block Term Decomposition Analysis in Long Segments of Atrial Fibrillation ECGs
}

Pedro Marinho R. de Oliveira, Vicente Zarzoso

\section{To cite this version:}

Pedro Marinho R. de Oliveira, Vicente Zarzoso. Block Term Decomposition Analysis in Long Segments of Atrial Fibrillation ECGs. XXXVI Simpósio Brasileiro de Telecomunicações e Processamento de Sinais, Sep 2018, Campina Grande, Brazil. 10.14209/sbrt.2018.182 hal-01949848

\section{HAL Id: hal-01949848 \\ https://hal.science/hal-01949848}

Submitted on 10 Dec 2018

HAL is a multi-disciplinary open access archive for the deposit and dissemination of scientific research documents, whether they are published or not. The documents may come from teaching and research institutions in France or abroad, or from public or private research centers.
L'archive ouverte pluridisciplinaire HAL, est destinée au dépôt et à la diffusion de documents scientifiques de niveau recherche, publiés ou non, émanant des établissements d'enseignement et de recherche français ou étrangers, des laboratoires publics ou privés. 


\title{
Block Term Decomposition Analysis in Long Segments of Atrial Fibrillation ECGs
}

\author{
Pedro Marinho R. de Oliveira and Vicente Zarzoso
}

\begin{abstract}
Responsible for $25 \%$ of strokes, atrial fibrillation (AF) is the most common sustained cardiac arrhythmia in clinical practice. A precise analysis of the atrial activity (AA) signal in electrocardiogram (ECG) recordings is necessary to better understand this challenging cardiac condition. Recently, the block term decomposition (BTD) has been proposed as a novel tool to extract AA in AF ECG signals noninvasively. However, this tensor factorization technique was performed only in short segments. In this paper, the BTD is assessed in long segments of an AF ECG, varying the observation window size. Experimental results show the performance of BTD in long segments of an AF ECG recording and the analysis of the observation window size. This promising tensor technique is compared to two matrix-based methods.
\end{abstract}

Keywords-Atrial Fibrillation, Block Term Decomposition, Electrocardiogram, Atrial Activity Extraction, Tensor Decomposition.

\section{INTRODUCTION}

Cardiac arrhythmia is a disease characterized by an irregular heartbeat. Especially affecting the eldery, atrial fibrillation (AF) is the most common sustained cardiac arrhythmia encountered in clinical practice, considered as the last great frontier of cardiac electrophysiology. Persistent AF represents a highly complex case of this arrhythmia, where extensive atrial remodelling has taken place due to sustained AF, significantly affecting atrial activity (AA) and AF perpetuation itself. The importance given to this challenging cardiac condition has increased in the past few years and is expected to increase even more, since it is becoming a major public health and economical concern, and its mechanisms are not completely understood. About 160000 new patients are diagnosed with AF every year only in USA and similar numbers are shown in European countries. By 2050, it is estimated that AF will become a new epidemic [2]. In addition, a patient with AF spends anually approximately $\$ 8700$ more in healthcare than a patient without AF. The treatment of this decease is estimated to add $\$ 26$ billion per year to the USA healthcare bill [1]. During a normal sinus rhythm in the electrocardiogram (ECG), the $\mathrm{P}$ wave corresponds to a normal atrial activation. During AF, the $\mathrm{P}$ wave is replaced by $\mathrm{f}$ waves (fibrillatory waves), which are present throughout the whole ECG recording. However, they are masked by the QRST complex that corresponds to the ventricular activity (VA) in each heartbeat

Signal processing techniques are useful and important tools in the analysis of cardiac signals. They are necessary to noninvasive extract the AA from the standard 12-lead ECG

Pedro Marinho R. de Oliveira and Vicente Zarzoso are with the Université Côte d'Azur, I3S Laboratory, CNRS, France. (e-mail: \{marinho, zarzoso\}@i3s.unice.fr). for an accurate analysis of the $\mathrm{f}$ waves, in order to better understand the mechanisms behind this disease. Matrix-based techniques to solve blind source separation (BSS) problems, such as principal component analysis (PCA) and independent component analysis (ICA) [3]-[5] have proved to be useful in AA extraction. However, matrix decompositions are known to have some limitations since constraints need to be imposed to guarantee uniqueness, e.g., orthogonality or statistical independence between the sources. Such constraints are mathematically coherent, but they may lack physiological grounds, making difficult the results interpretation.

Aiming to overcome the limitations imposed by matrix decompositions, a tensor factorization technique has been recently proposed to extract the AA signal from AF ECGs [6][9]. If compared to matrix-based techniques, tensor decompositions present some remarkable features like, for example, their essential uniqueness with minimal or no constraints. Another example is the fact that the rank of the tensor can exceed its dimensions, whereas in matrices the rank is limited by its lowest dimension. The block term decomposition (BTD) proposed in [13] suits the characteristics of AA in an AF signal, since atrial signals can be approximated by all-pole models and mapped onto Hankel matrices with rank equal to the number of poles [9]. The Hankel matrices containing the ECG data are stacked along the third dimension of a third-order tensor, and then processed by BTD. Previous experimental results in synthetic and real AF ECG data showed that BTD can outperform the matrix-based techniques for AA extraction in short segments of AF ECG recordings [6]-[8].

The performance of BTD depends considerably on its initialization and model parameters, especially the multilinear rank (the rank of the matrix factors) and the number of block terms of the tensor. In [9] an estimation method of the multilinear rank of BTD in an AF ECG tensor is proposed. This estimation is based on the application of three popular methods for the estimation of auto-regressive (AR) model order in the TQ segment of a heartbeat, where only AA is present. In addition, a sucessful AA extraction depends on the precise selection of the AA source among the others estimated sources. In [10] the BTD showed that the classical method of atrial source selection [3], [4] is not optimal, and then two suboptimal methods that provide better accuracy were proposed.

The BTD has proved to be useful extracting the AA signal from the AF ECG, being able to outperform the matrixbased techniques. However, this tensorial approach was only assessed in short segments of an AF ECG (1 to 5 seconds). Its performance in long segments remains an open question. To answer this question and analyze the observation window size on the performance of this new promising technique, the 
present work assesses BTD for the first time in long segments of a patient with persistent $\mathrm{AF}$ and compares it to two popular matrix-based methods for AA extraction: RobustICAf [11] and PCA [12]. Experimental results using Monte Carlo simulations evaluate the AA extraction performance of BTD on long segments of an AF ECG.

The rest of this paper is organized as follows. Section II introduces the notation used in the work, while Section III recalls the BTD as a tensor approach to solve BSS problems. Section IV discusses quantitative measures of AA content. Section V presents the database and the setup used in the experiments, whose results are reported in Section VI. Finally, Section VII gives an end to the paper with the conclusion of this work and the prospects of future works.

\section{NOTATIONS}

Scalars, vectors, matrices and tensors are represented by lower-case $(a, b, c, \ldots)$, boldface lower-case $(\mathbf{a}, \mathbf{b}, \mathbf{c}, \ldots)$, boldface capital $(\mathbf{A}, \mathbf{B}, \mathbf{C}, \ldots)$ and calligraphic $(\mathcal{A}, \mathcal{B}, \mathcal{C}, \ldots)$ letters, respectively.

The matrix transpose is represented by $(\cdot)^{T}$, symbol $\|\cdot\|$ represents the $l_{2}$-norm, $|\cdot|$ represents the absolute value and $\circ$ represents the outer product. $E[\cdot]$ denotes the mathematical expectation and the operator $\operatorname{diag}(\cdot)$ builds a diagonal matrix by placing its arguments along the diagonal. Given a thirdorder tensor $\mathcal{A} \in \mathbb{C}^{I_{1} \times I_{2} \times I_{3}}$, with scalars $a_{i_{1}, i_{2}, i_{3}}$, its frontal slices are represented by $\mathbf{A}_{. . i_{3}} \in \mathbb{C}^{I_{1} \times I_{2}}$. Given a matrix $\mathbf{A} \in \mathbb{C}^{I_{1} \times I_{2}}$, with scalar entries $a_{i_{1}, i_{2}}$, its $i_{1}^{\text {th }}$ row and the $i_{2}^{\text {th }}$ column are represented by $\mathbf{a}_{i_{1}}$, and $\mathbf{a}_{. i_{2}}$, respectively.

\section{BLOCK TERM DECOMPOSITION}

The BTD of an arbitrary third-order tensor $\mathcal{T} \in \mathbb{R}^{I_{1} \times I_{2} \times I_{3}}$ is written as

$$
\mathcal{T}=\sum_{r=1}^{R} \mathbf{E}_{r} \circ \mathbf{c}_{r}
$$

where $\mathbf{c}_{r} \in \mathbb{R}^{I_{3}}$ and $\mathbf{E}_{r} \in \mathbb{R}^{I_{1} \times I_{2}}$ has rank $L_{r}$ and admits a decomposition $\mathbf{E}_{r}=\mathbf{A}_{r} \mathbf{B}_{r}^{T}$, where $\mathbf{A}_{r} \in \mathbb{R}^{I_{1} \times L_{r}}$ and $\mathbf{B}_{r} \in$ $\mathbb{R}^{I_{2} \times L_{r}}$ have full column rank $L_{r}$. Equation (1) may then be rewritten as

$$
\mathcal{T}=\sum_{r=1}^{R}\left(\mathbf{A}_{r} \mathbf{B}_{r}^{T}\right) \circ \mathbf{c}_{r} .
$$

One can see that the BTD is a decomposition of $\mathcal{T}$ in multilinear rank- $\left(L_{r}, L_{r}, 1\right)$ terms. In [13, Theorem 2.2], it is shown that the BTD is essentially unique if the following conditions are satisfied:

1) The matrix factors $\mathbf{A}=\left[\begin{array}{llll}\mathbf{A}_{1} & \mathbf{A}_{2} & \ldots & \mathbf{A}_{R}\end{array}\right] \in$ $\mathbb{R}^{I_{1} \times \sum_{r=1}^{R} L_{r}}$ and $\mathbf{B}=\left[\begin{array}{llll}\mathbf{B}_{1} & \mathbf{B}_{2} & \ldots & \mathbf{B}_{R}\end{array}\right] \in$ $\mathbb{R}^{I_{2} \times \sum_{r=1}^{R} L_{r}}$ are full-column rank.

2) Matrix $\mathbf{C}=\left[\begin{array}{llll}\mathbf{c}_{1} & \mathbf{c}_{2} & \ldots & \mathbf{c}_{R}\end{array}\right] \in \mathbb{R}^{I_{3} \times R}$ does not contain proportional columns.

The first condition requires that $\sum_{r=1}^{R} L_{r} \leq I_{1}, I_{2}$. Milder uniqueness conditions are also presented in [13].

The ECG data matrix with $K$ leads and $N$ samples can be modeled as

$$
\mathbf{Y}=\mathbf{M S} \in \mathbb{R}^{K \times N}
$$

where $\mathbf{M} \in \mathbb{R}^{K \times R}$ is the mixing matrix, modeling the propagation of the cardiac electrical sources from the heart to the body surface, $\mathbf{S} \in \mathbb{R}^{R \times N}$ is the source matrix that contains the atrial, ventricular and noise sources, and $R$ is the number of sources [5]. Since the goal is to estimate $\mathbf{M}$ and $\mathbf{S}$ from the matrix $\mathbf{Y}$ (the only observed data), it is clear that AA extraction in an AF ECG recording is a BSS problem. In [13], the BTD is proposed as a solution of a BSS problem like (3). The idea to obtain a tensor from $\mathbf{Y}$ is to map its $k^{\text {th }}$ row onto a Hankel matrix $\mathbf{H}_{\mathbf{Y}}^{(k)} \in \mathbb{R}^{I \times J}$, where $I=J=\frac{N+1}{2}$ if $N$ is odd or $I=\frac{N}{2}$ and $J=\frac{N}{2}+1$ if $N$ is even, with

$$
\left[\mathbf{H}_{\mathbf{Y}}^{(k)}\right]_{i, j} \triangleq y_{k, i+j-1}
$$

where $i=1, \ldots, I, j=1, \ldots, J$, and $k=1, \ldots, K$. Next, the tensor is built by stacking each Hankel matrix along the third dimension (as frontal slices) of a third-order tensor $\mathcal{Y} \in \mathbb{R}^{I \times J \times K}$, that is

$$
\mathbf{Y}_{. . k}=\mathbf{H}_{\mathbf{Y}}^{(k)} .
$$

Tensor $\mathcal{Y}$ can be written in scalar form as

$$
y_{i, j, k}=\sum_{r=1}^{R} m_{k, r} s_{r, i+j-1} \text {. }
$$

The $k^{\text {th }}$ matrix slice of this tensor can be represented as

$$
\mathbf{Y}_{. . k}=\sum_{r=1}^{R} m_{k, r} \mathbf{H}_{\mathbf{S}}^{(r)}
$$

where $\mathbf{H}_{\mathbf{S}}^{(r)} \in \mathbb{R}^{I \times J}$ is a Hankel matrix built from the $r^{\text {th }}$ row of $\mathbf{S}$. For each $r$, the outer product between matrix $\mathbf{H}_{\mathbf{S}}^{(r)}$ and the $r^{\text {th }}$ column of $\mathbf{M}$, i.e., $\mathbf{m}_{. r}$, is being performed. This way, the tensor $\mathcal{Y}$ can be written as

$$
\mathcal{Y}=\sum_{r=1}^{R} \mathbf{H}_{\mathbf{S}}^{(r)} \circ \mathbf{m}_{. r} .
$$

Comparing Equations (1) and (8), it can be concluded that the ECG data tensor follows a BTD tensor model with the following correspondence

$$
\begin{aligned}
(\mathcal{T}) & \Longleftrightarrow(\mathcal{Y}) \\
\left(\mathbf{E}_{r}, \mathbf{c}_{r}\right) & \Longleftrightarrow\left(\mathbf{H}_{\mathbf{S}}^{(r)}, \mathbf{m}_{. r}\right) \\
\left(I_{1}, I_{2}, I_{3}, R\right) & \Longleftrightarrow(I, J, K, R) .
\end{aligned}
$$

Due to the quasi-periodic nature of AF, atrial sources can be represented by the exponential (or all-pole) model as

$$
s_{r, n}=\sum_{\ell=1}^{L_{r}} \lambda_{\ell, r} z_{\ell, r}^{n-1}
$$

where $n=1, \ldots, N, r=1, . ., R, L_{r}$ is the number of exponential terms, $z_{\ell, r}$ is the $\ell^{\text {th }}$ pole of the $r^{\text {th }}$ source, and $\lambda_{\ell, r}$ is the scaling coefficient. This way, their associated Hankel matrices accept the Vandermonde decomposition [14]:

$$
\mathbf{H}_{\mathbf{S}}^{(r)}=\mathbf{V}_{r} \operatorname{diag}\left(\lambda_{1, r}, \lambda_{2, r}, \ldots, \lambda_{L_{r}, r}\right) \hat{\mathbf{V}}_{r}^{T}
$$


with

$$
\mathbf{V}_{r}=\left[\begin{array}{cccc}
1 & 1 & \cdots & 1 \\
z_{1, r} & z_{2, r} & \cdots & z_{L_{r}, r} \\
\vdots & \vdots & \ddots & \vdots \\
z_{1, r}^{I-1} & z_{2, r}^{I-1} & \ldots & z_{L_{r}, r}^{I-1}
\end{array}\right] \in \mathbb{R}^{I \times L_{r}}
$$

and

$$
\hat{\mathbf{V}}_{r}=\left[\begin{array}{cccc}
1 & 1 & \ldots & 1 \\
z_{1, r} & z_{2, r} & \ldots & z_{L_{r}, r} \\
\vdots & \vdots & \ddots & \vdots \\
z_{1, r}^{J-1} & z_{2, r}^{J-1} & \ldots & z_{L_{r}, r}^{J-1}
\end{array}\right] \in \mathbb{R}^{J \times L_{r}} .
$$

In the case of different poles, the Vandermonde matrix with $L_{r} \leq I, J$ will have full-column rank $L_{r}$, so if $\mathbf{M}$ does not have proportional columns, the BTD in (8) will be essentially unique. In the case of equal poles, milder conditions can assure the uniqueness of (8) [13].

As previously described, BTD can outperform the matrix approaches regarding the AA extraction in short AF ECG recordings[6]-[9]. However, its performance in long AF ECG segments remains an open question, since, to the best of the authors' knowledge, the BTD has only been computed in short segments of AF ECGs.

\section{Atrial Activity Content Measurement}

Signal processing techniques used to solve BSS problems separate the original signal in several sources. In AF ECG, typically at least one of these sources contains the AA. However, it is unknown if the AA is concentrated only in a single source. In the present work, as in previous works, it is considered that the AA is concentrated in a single source, and the source with the most AA content is called the atrial source. In the frequency domain, the AA during AF has a peak between 3 and $9 \mathrm{~Hz}$. The position of this peak is called dominant frequency (DF). It is defined as potential atrial sources any source with DF placed in such an interval. Three parameters are used to measure AA extraction quality. In this work, the atrial source is selected by visual inspection helped by the parameters presented in this section. The first one is the spectral concentration (SC), that is, the relative amount of energy around the DF. The SC is computed as in [4]:

$$
S C=\frac{\sum_{f_{i}=0.82 f_{p}}^{1.17 f_{p}} P_{A A}\left(f_{i}\right)}{\sum_{f_{i}=0}^{F_{s} / 2} P_{A A}\left(f_{i}\right)}
$$

where $f_{p}$ is the value of the $\mathrm{DF}, F_{s}$ is the sampling frequency, $f_{i}$ is the discrete frequency and $P_{A A}$ is the power spectrum of the AA signal computed using Welch's method as in [4]. The second parameter used to better analyze the potential atrial sources is the kurtosis, denoted $K$, of the signal in the frequency domain acquired by a 4096-point FFT. As in [11], it is used the general expression of kurtosis valid for non-circular complex data, that is given by

$$
K=\frac{E\left[\left|\mathbf{s}_{r .}\right|^{4}\right]-2 E\left[\left|\mathbf{s}_{r .}\right|^{2}\right]^{2}-\left|E\left[\mathbf{s}_{r .}^{2}\right]\right|^{2}}{E\left[\left|\mathbf{s}_{r .}\right|^{2}\right]^{2}} .
$$

Kurtosis is a measure of peakedness and sparsity of a distribution and, when computed in the frequency domain, it naturally provides a quantitative measure of harmonicity of the signal. A high kurtosis in the frequency domain means that the power spectral density is sparse, which is suggestive of a harmonic signal like AA in AF. Also, kurtosis is parameter free, whereas SC depends on the DF and the definition of a suitable interval for interpretation [10]. The third parameter, used to eliminate sources with irrelevant content, is the power contribution to lead V1 given by

$$
P(r)=\frac{1}{N}\left\|m_{r}^{(V 1)} \mathbf{s}_{r .}\right\|^{2}
$$

in $\mathrm{mV}^{2}$, where $m_{r}^{(V 1)}$ is the contribution of the $r^{\text {th }}$ source to lead V1 (given by the corresponding element of the estimated mixing matrix) and $\mathbf{s}_{r}$. is the $r^{\text {th }}$ source, corresponding to the $r^{\text {th }}$ row of matrix $\mathbf{S}$ in Equation (3). The power contribution to lead V1 by an AA source is expected to be strong, since this lead is the one that typically reflects best AA in AF ECGs.

\section{Database And Experimental Setup}

Experiments on real AF ECG data evaluate the performance of BTD regarding the AA extraction in long segments varying the observation window size.

\section{A. Real AF ECG Data and Preprocessing}

Experiments are performed in 4 segments of a standard 12-lead ECG recording from a single patient suffering from persistent AF, considering all the 12 leads. These recordings belong to a database provided by the Cardiology Department of Princess Grace Hospital Center, Monaco. The recordings are acquired at a $977 \mathrm{~Hz}$ sampling rate and are preprocessed by a zero-phase forward-backward type-II Chebyshev bandpass filter with cutoff frequencies of 0.5 and $40 \mathrm{~Hz}$, in order to suppress high-frequency noise and baseline wandering. A $15-$ second segment in lead II from the patient is shown on Figure 1. The segments have about 2.5, 5, 10 and 15 seconds and they all have the same starting sample. They are downsampled by a factor of $4,8,16$ and 24 , respectively, since the originally built third-order tensors pose some difficulties to Tensorlab MATLAB toolbox [15].

\section{B. BTD Setup}

The BTD is implemented using the non-linear least squares (NLS) method available in Tensorlab choosing $R=12$ and $L_{r}=95$, for all $r=1,2, \ldots, R$. This choice is made based on the work [9], that shows that such values provide satisfactory results for the heartbeat with the largest TQ segment of the patient considered in the present work. The tolerance threshold for BTD convergence is set to $10^{-9}$ and the maximum number of iterations is set to 1000 . Ten Monte Carlo runs, with Gaussian random initialization for the matrix and vector factors at each run, are used to analyze the performance of BTD in the AF patient regarding AA signal extraction. Monte Carlo runs are needed, since BTD has a strong dependence on its initialization. This tensorial technique is compared to the matrix-based methods PCA and RobustICA-f. 


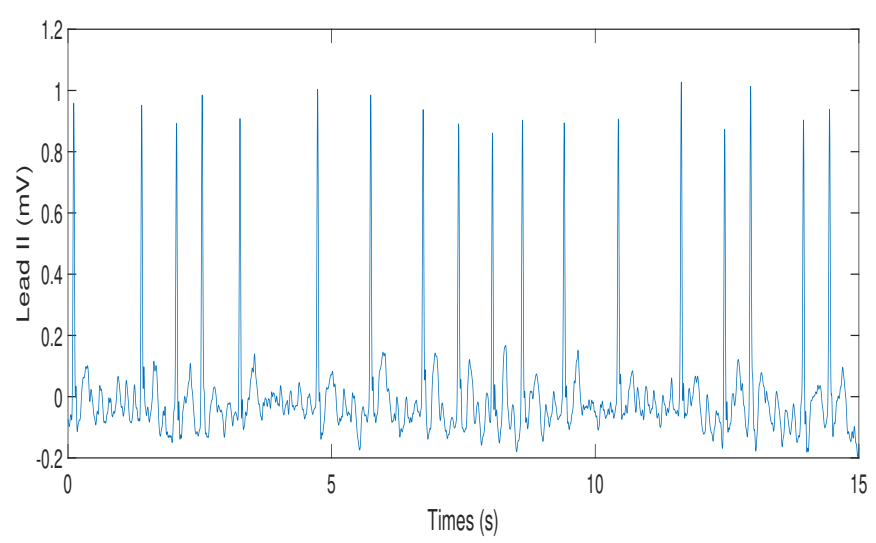

Fig. 1: A 15-second segment of an AF ECG recording from one patient. Only bipolar limb lead II is shown, for clarity, although the full 12 leads are processed.

TABLE I: Values of SC (\%) for PCA and RobustICA-f. For $\mathrm{BTD}$, the maximum $\left(\mathbf{B T D}_{\max }\right)$ and the mean $\left(\mathbf{B T D}_{\text {mean }}\right)$ values of SC (\%) of ten independent runs is shown.

\begin{tabular}{|c|c|c|c|c|}
\hline & BTD $_{\max }$ & BTD $_{\text {mean }}$ & PCA & RobustICA-f \\
\hline $\mathbf{2 . 5} \mathbf{~ s}$ & 94.04 & 74.03 & 55.29 & 69.08 \\
\hline $\mathbf{5} \mathbf{~ s}$ & 77.88 & 65.56 & 49.03 & 66.13 \\
\hline $\mathbf{1 0} \mathbf{~ s}$ & 90.93 & 73.49 & 56.40 & 72.22 \\
\hline $\mathbf{1 5} \mathbf{~ s}$ & 93.01 & 77.87 & 54.36 & 71.80 \\
\hline
\end{tabular}

\section{Results}

\section{A. Long Segments Performance}

Table I shows the values of SC in \% for PCA and RobustICA-f. The mean of the ten independent runs and the maximum value is shown for BTD. It can be seen in Table I that the mean of the SC for BTD is very close to the matrix-based techniques, outperforming PCA in all the observed segments and RobustICA-f in 3 out of the 4 segments with different lengths. Also, BTD is superior for all observed segments in 5 to 8 out of the 10 independent runs, showing that it can easily have superior performance if the right model parameters $\left(R, L_{r}\right)$ and initialization are chosen. It can be seen also that the maximum SC presents a high value $(\geq 75 \%)$ for all the observed segments, and in all cases, superior to that of matrix techniques, meaning that, with the right initialization, BTD outperformed to the matrix-based techniques for the considered window lengths.

In the observed segments of different lengths, BTD finds more potential atrial sources than PCA and RobustICA-f. Discovering many potential atrial sources may be an interesting outcome, since it increases the possibility of finding some features that, although weakly contributing to the AA, may provide useful physiological and clinical information about the arrhythmia.

Figures 2 and 3 illustrate the estimated atrial source by the three BSS techniques compared in this paper, in the time and frequency domain, respectively, for the best performance of BTD in the 15-second segment. These two figures show the satisfactory performance of BTD in estimating the AA in long segments of an AF ECG, as well as its superiority compared

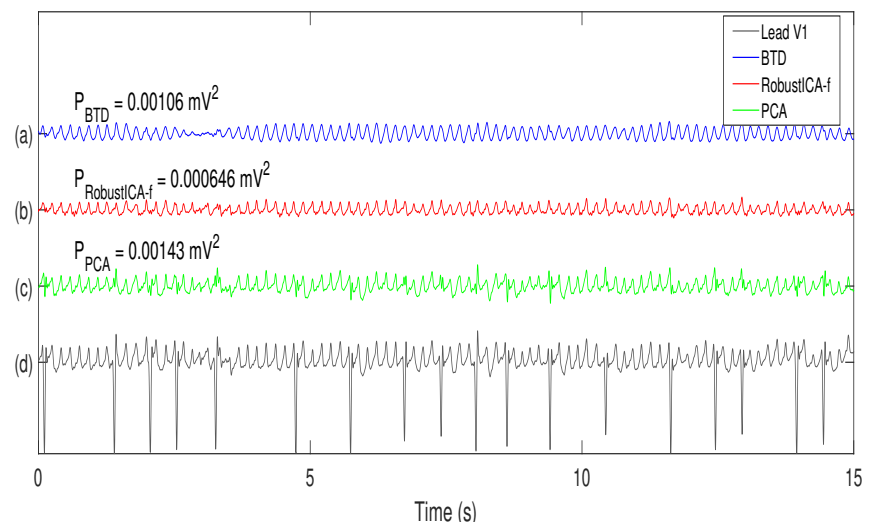

Fig. 2: Estimated atrial sources contribution to lead V1 in the 15-second segment by BTD, RobustICA-f and PCA in the time domain (in $\mathrm{mV}$ ). AA signal estimations are vertically shifted for clarity.

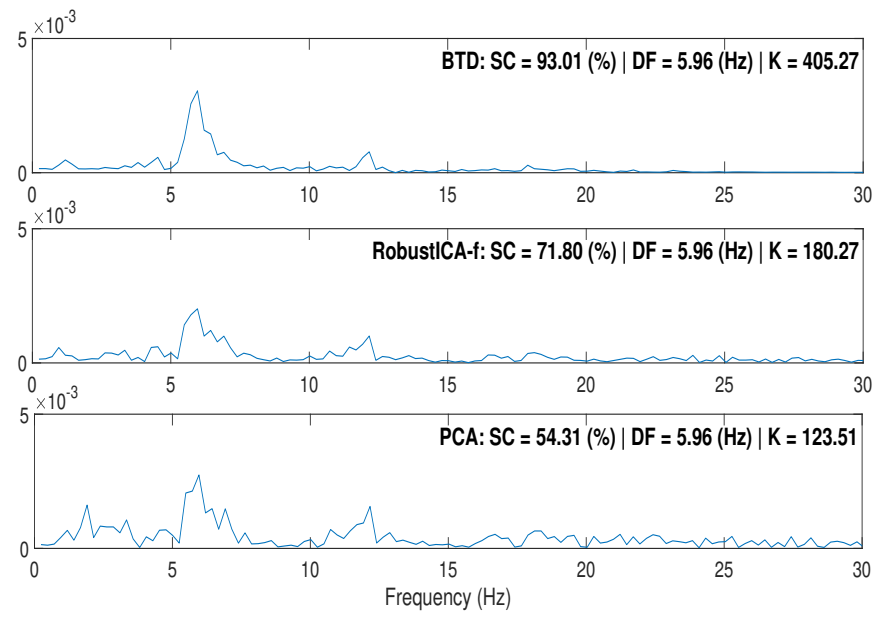

Fig. 3: Atrial sources contribution to lead V1 in the 15-second segment estimated by BTD, RobustICA-f and PCA in the frequency domain (in $\mathrm{mV} / \sqrt{\mathrm{Hz}}$ ).

to the matrix-based methods, as quantified by the higher SC and kurtosis values.

\section{B. Observation Window Size Analysis}

The observation window size used in the experiments extends from 2.5 to 15 seconds, in order to analyze its influence on BTD performance. Figures 4 and 5 show the variation of SC and kurtosis, respectively, over the 10 independent runs for all the window observation sizes analyzed in this work. This variation is illustrated by a box-and-whisker plot, where the red lines represent the median and the blue boxes the percentiles. Due to the lack of space, similar figures for PCA and RobustICA-f are not shown in this paper.

It can be seen that there exists a certain variation of SC and kurtosis over the runs, for each observation window size. This is expected, since BTD performance depends considerably on the initialization of its model factors. However, there is no clear trend in the computed parameters, which shows that the influence of the observation window size on the performance of BTD is not very significant. 


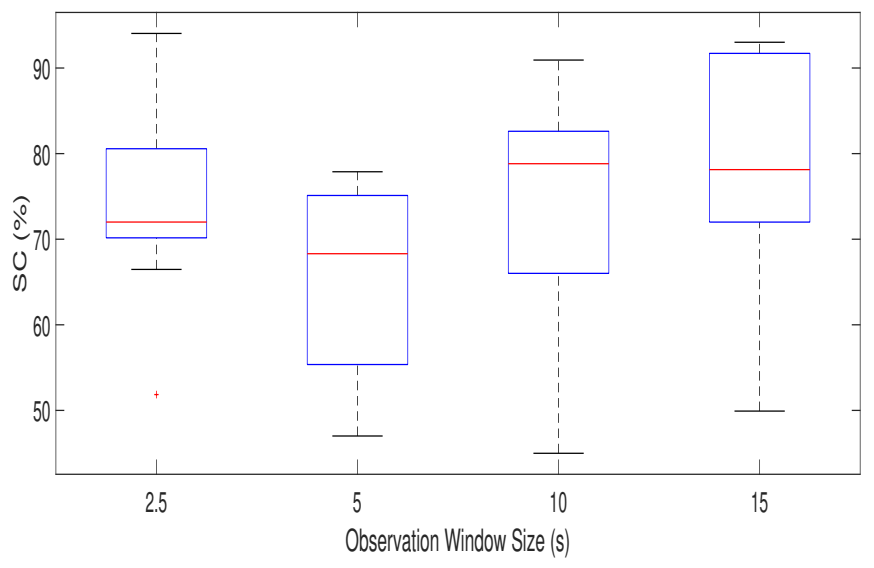

Fig. 4: SC $(\%)$ of the AA signals estimated by BTD from the observed AF ECG segments over ten independent runs.

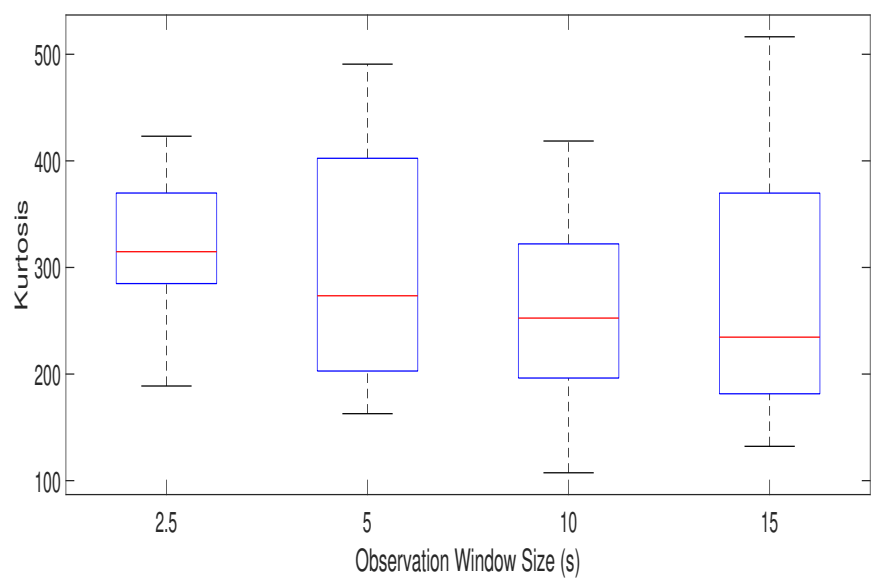

Fig. 5: Kurtosis of the AA signals estimated by BTD from the observed AF ECG segments over ten independent runs.

The only drawback observed when processing long segments of ECG using BTD is the fact that the original segment must be downsampled by high factors, since Tensorlab has some difficulties in processing a tensor with large dimensions. Downsampling by a high factor could cause some loss of information in the signal.

\section{CONCLUSION}

$\mathrm{AF}$ is becoming a major public health and economical concern. Signal processing techniques to solve BSS problems arise as a key tool to better understand the mechanisms behind this challenging cardiac condition. Aiming to overcome the limitations of matrix-based methods, a tensorial technique has been recently proposed as a useful tool for atrial signal extraction in AF ECGs. The first contribution of the present work is the analysis of BTD in time segments with varying length of an AF ECG, showing that this tensor technique presents a satisfactory performance not only in short segments $(2.5 \mathrm{~s})$, but also in long segments (15 s). The second contribution of the present work is the analysis of the observation window size on the performance of BTD. The choice of the size of the observation window is shown to be not very significant regarding the quality of the atrial signal extracted. The only drawback observed is the need of downsampling the long segment by a high factor, which may cause some loss of information. Future works would aim at analyzing the temporal (or intra-patient) stability of BTD. Experiments should be performed in a large database of $\mathrm{AF}$ patients to validate these results.

\section{ACKNOWLEDGEMENTS}

Pedro Marinho is funded by a PhD scholarship from the IT Doctoral School of the Universite Côte d'Azur. Vicente Zarzoso is a member of the Institut Universitaire de France.

\section{REFERENCES}

[1] C. T. January, L. S. Wann, J. S. Alpert, H. Calkins, J. C. Cleveland, J. E. Cigarroa, J. B. Conti, et al., "2014 AHA/ACC/HRS guideline for the management of patients with atrial fibrillation: A report of the American College of Cardiology/American Heart Association Task Force on practice guidelines and the Heart Rhythm Society", Circulation, vol. 64, no. 21, pp. 2246-2280, Dec. 2014.

[2] L. Mainardi, L. Sörnmo, S. Cerutti, "Understanding Atrial Fibrilation: The Signal Processing Contribution", Synthesis Lectures on Biomedical Engineering, Morgan \& Claypool Publishers, 2008.

[3] J. J. Rieta, F. Castells, C. Sánchez, V. Zarzoso, and J. Millet, "Atrial activity extraction for atrial fibrillation analysis using blind source separation”, IEEE Transactions on Biomedical Engineering, vol. 51, no. 7, pp. 1176-1186, Jul. 2004.

[4] F. Castells, J. J. Rieta, J. Millet, and V. Zarzoso, "Spatiotemporal blind source separation approach to atrial activity estimation in atrial tachyarrhythmias", IEEE Transactions on Biomedical Engineering, vol. 52, no. 2, pp. 258-267, Feb. 2005.

[5] V. Zarzoso, "Extraction of ECG characteristics using source separation techniques: exploiting statistical independence and beyond", Advanced Biosignal Processing, A. Naït-Ali, Ed. Berlin, Heidelberg: Springer Verlag, 2009, ch. 2, pp. 15-47.

[6] L. N. Ribeiro, A. R. Hidalgo-Muñoz, and V. Zarzoso, "Atrial signal extraction in atrial fibrillation electrocardiograms using a tensor decomposition approach", in Proc. EMBC-2015, 37th Annual International Conference of the IEEE Engineering in Medicine and Biology Society, Milan, Italy, Aug. 25-29, 2015, pp. 6987-6990.

[7] L. N. Ribeiro, A. R. Hidalgo-Muñoz, G. Favier, J. C. M. Mota, A. L. F. de Almeida, and V. Zarzoso, "A tensor decomposition approach to noninvasive atrial activity extraction in atrial fibrillation ECG", in Proc. EUSIPCO-2015, XXIII European Signal Processing Conference, Nice, France, Aug. 31-Sept. 4, 2015, pp. 2576-2580.

[8] L. N. Ribeiro, A. L. F. de Almeida, and V. Zarzoso, "Enhanced block term decomposition for atrial activity extraction in atrial fibrillation ECG", in Proc. SAM-2016, 9th IEEE Sensor Array and Multichannel Signal Processing Workshop, Rio de Janeiro, Brazil, July 10-13, 2016.

[9] V. Zarzoso, "Parameter estimation in block term decomposition for noninvasive atrial fibrillation analysis", in Proc. CAMSAP-2017, IEEE International Workshop on Computational Advances in Multi-Sensor Adaptive Processing, Curaçao, Dutch Antilles, Dec. 10-13, 2017.

[10] P. M. R. de Oliveira and V. Zarzoso, "Source analysis and selection using block term decomposition in atrial fibrillation", in Proc. LVA/ICA-2018, 14th International Conference on Latent Variable Analysis and Signal Separation, Guildford, United Kingdom, Jul. 2-6, 2018, pp. 46-56.

[11] V. Zarzoso and P. Comon, "Robust independent component analysis by iterative maximization of the kurtosis contrast with algebraic optimal step size", IEEE Transactions on Neural Networks, vol. 21, no. 2, pp. 248-261, 2010

[12] I. Jolliffe, Principal Component Analysis, Wiley Online Library, 2005.

[13] L. De Lathauwer, "Blind separation of exponential polynomials and the decomposition of a tensor in rank- $\left(l_{r}, l_{r}, 1\right)$ terms", SIAM Journal on Matrix Analysis and Applications, vol. 32, no. 4, pp. 1451-1474, 2011.

[14] D. L. Boley, F. T. Luk, and D. Vandevoorde, "Vandermonde factorization of a Hankel matrix", in Proceedings of the Workshop on Scientific Computing, Hong Kong, March 1997.

[15] N. Vervliet, O. Debals, L. Sorber, M. Van Barel, and L. De Lathauwer, Tensorlab 3.0, Available online, Mar. 2016. URL: https://www.tensorlab.net/ 